\title{
A type system for Continuation Calculus
}

\author{
Herman Geuvers \\ Radboud University Nijmegen, Technical University Eindhoven, the Netherlands \\ Wouter Geraedts \\ Bram Geron \\ Radboud University Nijmegen, the Netherlands $\quad$ School of Computer Science, University of Birmingham, UK \\ Judith van Stegeren \\ Radboud University Nijmegen, the Netherlands
}

\begin{abstract}
Continuation Calculus (CC), introduced by Geron and Geuvers [2], is a simple foundational model for functional computation. It is closely related to lambda calculus and term rewriting, but it has no variable binding and no pattern matching. It is Turing complete and evaluation is deterministic. Notions like "call-by-value" and "call-by-name" computation are available by choosing appropriate function definitions: e.g. there is a call-by-value and a call-by-name addition function.

In the present paper we extend $\mathrm{CC}$ with types, to be able to define data types in a canonical way, and functions over these data types, defined by iteration. Data type definitions follow the so-called "Scott encoding" of data, as opposed to the more familiar "Church encoding".

The iteration scheme comes in two flavors: a call-by-value and a call-by-name iteration scheme. The call-by-value variant is a double negation variant of call-by-name iteration. The double negation translation allows to move between call-by-name and call-by-value.
\end{abstract}

\section{Introduction}

Continuation calculus (or CC) [2] is a crossover between term rewriting systems and $\lambda$-calculus. Rather than focusing on expressions, continuation calculus treats continuations as its fundamental object. This is accomplished by restricting evaluation to strictly top-level, discarding the need for evaluation inside contexts. This also fixes an evaluation order, so the representation of a program in CC depends on whether call-by-value or call-by-name is desired. Furthermore, CC "separates code from data" by placing the former in a static program, which is sourced for reductions on a term. Variables are absent from terms, and no substitution happens inside terms.

Despite the obvious differences between CC and $\lambda$-calculus with continuations (or $\lambda_{C}$ ), there seems to be a strong correspondence. For instance, it has been suggested [3] that programs in either can be simulated in the other up to (non)termination, in an untyped setting. Progress so far suggests that continuation calculus might become a useful alternative characterization of $\lambda_{C}$, and theorems in one system could apply without much effort to the other.

The purpose of this paper is to strengthen the correspondence between $\mathrm{CC}$ and $\lambda$-calculus, by introducing a type system for CC and by showing how data types and functions over data can be defined in CC. The type system rejects some undesired terms and the types emphasize the difference between call-byname and call-by-value. Also, the types pave the way for proving properties of the programs. The types themselves do not enforce termination in general, because the system is 'open': programs are

Paulo Oliva (Ed.): Classical Logic and Computation 2014

EPTCS 164, 2014, pp. 1-17 doi 10.4204/EPTCS.164.1 
understood to be only parts of a larger whole, and names with no rule in a certain program are names whose "behavior" is left unspecified. However, if the programs are defined using only iteration and non-circular program rules, all terms are terminating. This we show in a separate paper.

\subsection{Informal definition of $\mathrm{CC}$}

Terms in CC are of the shape $n \cdot t_{1} \cdot t_{2} \ldots . . t_{k}$, where $n$ is a name and $t_{i}$ is again a term. The 'dot' denotes binary application, which is left-associative. In CC, terms can be evaluated by applying program rules which are of the shape

$$
n \cdot x_{1} \cdot x_{2} \ldots . x_{p} \longrightarrow u,(*)
$$

where $u$ is a term over variables $x_{1} \ldots x_{p}$. However, this rule can only be applied on the 'top level':

- reduction is not a congruence;

- rule $(*)$ can only be applied to the term $n \cdot t_{1} \cdot t_{2} \ldots . t_{k}$ in case $k=p$,

- then this term evaluates to $u\left[t_{1} / x_{1}, \ldots, t_{p} / x_{p}\right]$.

$\mathrm{CC}$ has no pattern matching or variable binding, but it is Turing complete and a translation to and from the untyped $\lambda$-calculus can be defined that preserves and reflects termination, see [3].

In continuation calculus, the natural numbers are represented by the names Zero and Succ and the following two program-rules:

$$
\begin{array}{lll}
\text { Zero. } c_{1} \cdot c_{2} & \longrightarrow & c_{1} \\
\text { Succ. } x \cdot c_{1} \cdot c_{2} & \longrightarrow & c_{2} \cdot x
\end{array}
$$

So Zero represents 0, Succ.Zero represents 1, Succ.(Succ.Zero) represents 2 etcetera. This representation of data follows the so-called Scott encoding, which is known from the untyped lambda calculus by defining Zero $:=\lambda x y . x$, Succ $:=\lambda n . \lambda x y . y n$ (e.g. see [1,5]). The Scott numerals have "case-distinction" built in (distinguishing between 0 and $n+1$ ), which can be used to mimic pattern matching. The more familiar Church numerals have iteration built in. For Scott numerals, iteration has to be added, or it can be obtained from the fixed-point combinator in the case of untyped lambda calculus. For CC the situation is similar: we have to add iteration ourselves.

As an example, we define addition in two ways: in call-by-value (CBV) and in call-by-name (CBN) style ( [2]).

\section{Example 1.1}

$$
\begin{aligned}
& \text { AddCBV.n.m.c } \longrightarrow \text { n.(c.m).(AddCBV'.m.c) } \\
& \operatorname{AddCBV}^{\prime} . m . c . n^{\prime} \longrightarrow \operatorname{AddCBV} \cdot n^{\prime} .(\text { Succ. } m) . c \\
& \operatorname{AddCBN} \cdot n \cdot m \cdot c_{1} \cdot c_{2} \longrightarrow n \cdot\left(m \cdot c_{1} \cdot c_{2}\right) \cdot\left(\operatorname{AddCBN}^{\prime} \cdot m \cdot c_{2}\right) \\
& \operatorname{AddCBN}^{\prime} \cdot m \cdot c_{2} \cdot n^{\prime} \longrightarrow c_{2} \cdot\left(\operatorname{AddCBN} \cdot n^{\prime} \cdot m\right)
\end{aligned}
$$

For AddCBV we find that AddCBV.(Succ ${ }^{n}$.Zero).(Succ ${ }^{m}$.Zero).c evaluates to $c$. (Succ ${ }^{n+m}$.Zero): the result of the addition function is computed completely and passed as argument to the continuation $c$. For AddCBN, only a first step in the computation is carried out and then the result is passed to the appropriate continuation $c_{1}$ or $c_{2}$. 
Continuation calculus as it occurs in [2] is untyped. In the present work we present a typing system for continuation calculus. The typing system gives the user some guarantee about the meaning and wellformedness of well-typed terms. We also develop a general procedure for defining algebraic data-types as types in $\mathrm{CC}$ and for transforming functions defined over these data types into valid typed terms in CC. In a separate paper we prove termination of all well-formed iterative CC programs [4].

\section{Formal definition of $\mathrm{CC}$}

For the detailed formal definition, we refer to [2]. Here we give a short recap of CC. The terms are either a name, or the combination $t . u$ of two terms $t$ and $u ; \mathscr{N}$ is any infinite set of names. So, the terms do not contain variables. (One could add them, but it's not necessary.) Names act as labels for functions and constructors in CC. Names in CC start with an uppercase letter and are printed in bold. The dot is left-associative, so we write $\left(\left(\left(n . t_{1}\right) . t_{2}\right) \ldots . t_{k}\right)$ as $n . t_{1} \ldots . t_{k}$.

The head of a term is its 'leftmost' name: head $\left(n . t_{1} . t_{2} \ldots . . t_{k}\right)=n$. The length of a term is the number of dots towards the head: length $\left(n \cdot t_{1} \cdot t_{2} \ldots . t_{k}\right)=k$.

To define programs we assume any infinite set $\mathscr{V}$ of variables. A program is a set of rules, each of the following shape

$$
n \cdot x_{1} \cdot x_{2} \ldots . x_{k} \longrightarrow u
$$

where the $x_{i}$ are distinct variables and $u$ is a term over the variables $x_{1}, \ldots, x_{k}$, so $u$ is a term that may use, apart from names, also the variables $x_{1}, \ldots, x_{k}$. We say that the rule defines the name $n$. Within a program, a name may occur at most once as the head of a rule. If $P$ is a program, the domain of $P$, $\operatorname{dom}(P)$ is the set of names that is defined in $P$.

Let a program $P$ be given. A term can be evaluated in $P$ by applying one of the rules of $P$ to the whole term as follows. Suppose $P$ contains the rule $n . x_{1} \cdot x_{2} \ldots x_{k} \longrightarrow u$, then

$$
n . t_{1} \cdot t_{2} \ldots . t_{k} \longrightarrow_{P} u\left[t_{1} / x_{1}, \ldots, t_{k} / x_{k}\right]
$$

where the latter denotes the substitution of $t_{1}, \ldots, t_{k}$ for $x_{1}, \ldots, x_{k}$. We usually omit the subscript $P$ and just write $\longrightarrow$, as $P$ will be clear from the context.

It should be noted that one does not evaluate 'under the application'. To make this explicit we introduce some more terminology. A name $n$ has arity $k$ in $P$ if $P$ contains a rule of the form

$$
n \cdot x_{1} \cdot x_{2} \ldots . x_{k} \longrightarrow u
$$

Similarly, a term $t$ has an arity in $P$ if $\operatorname{arity}($ head $(t)) \geq$ length $(t)$ and we define

$$
\operatorname{arity}(t):=\operatorname{arity}(\operatorname{head}(t))-\text { length }(t)
$$

A term $t$ is defined in $P$ if head $(t) \in \operatorname{dom}(P)$. Otherwise $t$ is undefined in $P$. A defined term is either complete, if arity $(t)=0$, or incomplete if $\operatorname{arity}(t)>0$, or invalid if it has no arity.

We write $A \longrightarrow B$ for " $B$ is a reduct of $A$ " and $A \longrightarrow B$ for " $A$ reduces in zero or more steps to $B$ ". Because every name is defined at most once in the set of program rules, every program is a deterministic rewriting system. 
A term $M$ is said to be terminating (or strongly normalizing) in $P$ if there exists a reduct $N$ such that $N$ can no longer be rewritten using the rules from $P$. Then $N$ can be undefined, incomplete, or invalid. We write

$$
\begin{array}{ll}
M \downarrow_{P} & \text { if } M \text { can not be rewritten using the rules of } P \\
M \downarrow_{P} & \text { if } \exists N\left(M \longrightarrow N \wedge N \downarrow_{P}\right)
\end{array}
$$

Note that $\mathrm{M} \downarrow_{P}$ implies $M \longrightarrow N \downarrow_{P}$, as $M \longrightarrow N$ can mean that $M$ rewrites to $N$ in zero steps.

The simplest notion of equality between terms in $\mathrm{CC}$ is the transitive, symmetric, reflexive closure of $\longrightarrow$, which we denote by $=_{P}$. So $M_{1}={ }_{P} M_{2}$ in case there is an $N$ such that $M_{1} \longrightarrow_{P} N$ and $M_{2} \longrightarrow_{P} N$. This is an interesting equivalence relation, however, it is much too fine, as we show in the following example. (See also [2].)

Example 2.1 For the call-by-name addition of Example1.1] we find that

$$
\text { AddCBN.(Succ.Zero).Zero. } c_{1} \cdot c_{2} \longrightarrow \text { AddCBN' }^{\prime} \text { ZZero. } c_{2} . \text { Zero } \longrightarrow c_{2} .(\text { AddCBN.Zero.Zero) }
$$

If we also compute Succ.Zero. $c_{1} . c_{2}$, we obtain $c_{2}$. Zero, which is not the same term, so we don't have AddCBN.(Succ.Zero).Zero. $c_{1} \cdot c_{2}={ }_{P}$ Succ.Zero. $c_{1} \cdot c_{2}$.

If we also allow computing 'under the function $c_{2}$ ', the terms are still not equal: AddCBN.Zero.Zero does not reduce to Zero. However, when supplied with two continuations, $d_{1}$ and $d_{2}$, they are equal: AddCBN.Zero.Zero. $d_{1} \cdot d_{2} \longrightarrow d_{1}$ and Zero. $d_{1} . d_{2} \longrightarrow d_{1}$.

In the example we see two terms $M$ and $N$ which are 'equal for all practical purposes', but we don't have $M={ }_{P} N$. We say that two terms $M$ and $N$ are observationally equivalent under program $P$, notation $M \approx_{P} N$, if for all extension programs $P^{\prime} \supseteq P$ and all terms $\mathrm{X}$

$$
X . M \downarrow_{P^{\prime}} \Longleftrightarrow X . N \downarrow_{P^{\prime}}
$$

We recall some properties about $\approx_{P}$ from [2]. Proofs can be found in [2].

Lemma 2.2 The relation $\approx_{P}$ is a congruence, that is, if $M_{1} \approx_{P} M_{2}$ and $N_{1} \approx_{P} N_{2}$, then $M_{1} \cdot N_{1} \approx_{P} M_{2} \cdot N_{2}$.

Lemma 2.3 Let $M, N$ be terms of arity $k$. If $M . c_{1} \ldots . c_{k}={ }_{P} N . c_{1} \ldots . c_{k}$ for fresh names $c_{1}, \ldots, c_{k}$, then $M \approx_{P} N$.

Corollary 2.4 If $M={ }_{P} N$ and $\operatorname{arity}(M)=\operatorname{arity}(N)=0$, then $M \approx_{P} N$.

It is not in general the case that $M={ }_{P} N$ implies $M \approx_{P} N$. The reason is that reduction of a term need not respect the arity, giving rise to undesired situations, as can be seen in the following example (also taken from [2]). Our typing system will prevent these situations to occur.

Example 2.5 Consider the following program rules

$$
\begin{aligned}
\text { Id } . x & \longrightarrow x \\
\text { Omega } x & \longrightarrow x \cdot x
\end{aligned}
$$

Then Id.Omega $\longrightarrow$ Omega, which is an incomplete term. If we append another term to Id.Omega, it becomes invalid: Id.Omega. $M$ has no arity. On the other hand, Omega.Omega $\longrightarrow$ Omega.Omega, so this term is non-terminating. Hence, Id.Omega $\longrightarrow$ Omega, but Id.Omega $\varkappa_{P}$ Omega. 
The type system will prevent situations as in Example 2.5, by making the program rule for Omega not 'well-typed' (and thereby not allowed). Also note that Id.Omega $\not_{P}$ Omega is only possible because $\operatorname{arity}($ Id.Omega $) \neq \operatorname{arity}($ Omega $)$. The type system will make sure that, if $M \longrightarrow N$, then $N$ also has arity 0 .

\section{Types for Continuation Calculus}

Definition 3.1 We define types of CC as follows.

$$
\text { Type }:=\perp \mid \text { Var } \mid(\text { Type } \rightarrow \text { Type }) \mid \mu \text { Var.Type. }
$$

where, in $\mu X . \Phi$, we require $\Phi$ to be of the shape

$$
\sigma^{1} \rightarrow \ldots \rightarrow \sigma^{n} \rightarrow \perp(\text { with } n \geq 0),
$$

with each $\sigma^{i}$ of the shape

$$
\left.\tau_{1}^{i} \rightarrow \ldots \rightarrow \tau_{a_{i}}^{i} \rightarrow \perp \text { (with } a_{i} \geq 0\right),
$$

where each $\tau_{j}^{i}$ is either $X$ or does not contain $X$.

As usual, we leave out the parentheses around functions types, so $A \rightarrow B \rightarrow C$ always means $A \rightarrow(B \rightarrow$ $C)$.

As a consequence of the above definition, if we have a type $\mu X . \Phi(X)$, then $X$ occurs positively in $\Phi$. We could have been more liberal, by allowing all types $\mu X . \Phi(X)$ where $X$ occurs positively in $\Phi(X)$, but that is unnecessary to interpret first order data-types.

The intention of the recursive type $\mu X . \Phi(X)$ is that it denotes the type $\sigma$ for which $\sigma=\Phi(\sigma)$. To give the $\mu$-types their semantics, we introduce type equalities.

Definition 3.2 We define equality between types, $\sigma=\tau$, as the least equivalence relation that can be derived using the following rules.

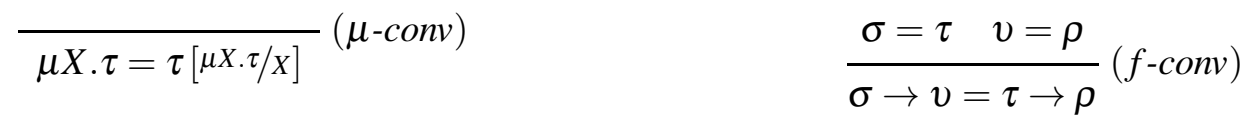

For a program rule $n . x_{1} \ldots x_{n} \longrightarrow u$ with $x_{1}: \tau_{1}, \ldots, x_{k}: \tau_{k}$, we will define $n$ to have the following type: $n: \tau_{1} \rightarrow \ldots \rightarrow \tau_{k} \rightarrow \perp$. So $\perp$ will be used as the type of complete CC-terms. This is very much in line with the approach taken by Miquel [7].

CC-types will be printed in bold. For example, the type representing natural numbers $\mathbb{N}$ will be printed as Nat. Abstract types (i.e. for any $\sigma \in$ Type) are printed as $\sigma, \tau, A, B$, etc.

Example 3.3 The types in CC of some well-known algebraic data-types

$$
\begin{array}{ll}
\text { Bool } & :=\perp \rightarrow \perp \rightarrow \perp \\
\text { Nat } & :=\mu T \cdot \perp \rightarrow(T \rightarrow \perp) \rightarrow \\
\text { List }_{A} & :=\mu T . \perp \rightarrow(A \rightarrow T \rightarrow \perp) \rightarrow \perp \\
\text { BinTree }_{A} & :=\mu T . \perp \rightarrow(A \rightarrow T \rightarrow T \rightarrow \perp) \rightarrow \perp
\end{array}
$$


Convention 3.4 We make use of the convention in logic to define $\neg A$ as $A \rightarrow \perp$ to introduce $\neg \sigma$ as an abbreviation for the type $\sigma \rightarrow \perp$. Similarly, $\neg \neg \sigma$ denotes $(\sigma \rightarrow \perp) \rightarrow \perp$.

Definition 3.5 A program signature $\Sigma$ is a finite set $\mathscr{N} \times$ Type

$$
\Sigma=n_{1}: \sigma_{1}, \ldots, n_{p}: \sigma_{p} \quad\left(\text { with all } n_{i} \text { distinct }\right)
$$

A typing context $\Gamma$ is a finite set $\mathscr{V} \times$ Type:

$$
\Gamma=x_{1}: \sigma_{1}, \ldots, x_{n}: \sigma_{n} \quad \text { (with all } x_{i} \text { distinct) }
$$

The signature gives the types of the names; it is constructed specifically for a program $P$. The context is just a "temporary" set of variables; contexts will be used to define program rules.

We are interested in two kinds of judgment:

1. $\Sigma \vdash P$, a program judgment, to express that, given a program signature $\Sigma, P$ is a well-typed program. So $P$ will consist of program rules.

2. $\Gamma \vdash_{\Sigma} M: A$, a typing judgment, to express that $M-$ a term with free variables in $\Gamma-$ has type $A$, given program signature $\Sigma$ and typing context $\Gamma$.

Definition 3.6 The derivation rules to derive typing judgments are the following

$$
\begin{aligned}
& \text { (Var) } \frac{x: \sigma \in \Gamma}{\Gamma \vdash_{\Sigma} x: \sigma} \quad \text { (Name) } \frac{n: \sigma \in \Sigma}{\Gamma \vdash_{\Sigma} n: \sigma} \\
& \text { (Appl) } \frac{\Gamma \vdash_{\Sigma} M: \sigma \rightarrow \tau \quad \Gamma \vdash_{\Sigma} N: \sigma}{\Gamma \vdash_{\Sigma} M . N: \tau} \quad(=- \text { conv }) \quad \frac{\Gamma \vdash_{\Sigma} M: \sigma \quad \sigma=\tau}{\Gamma \vdash_{\Sigma} M: \tau}
\end{aligned}
$$

Definition 3.7 The derivation rules to derive program judgments are the following

(Nil) $\quad \overline{\Sigma \vdash \emptyset}$

(Cons) $\frac{\Sigma \vdash P \quad x_{1}: A_{1}, \ldots, x_{k}: A_{k} \vdash_{\Sigma} q: \perp n: A_{1} \rightarrow \ldots \rightarrow A_{k} \rightarrow \perp \in \Sigma}{\Sigma \vdash P \cup\left\{n \cdot x_{1} \ldots x_{k} \longrightarrow q\right\}}$ if $n$ not defined in $P$

We say that program $P$ is well-typed in $\Sigma$ in case $\Sigma \vdash P$. Usually, $\Sigma$ will be clear and we just say that $P$ is well-typed. Similarly, we say that the program rule $n . x_{1} \ldots x_{k} \longrightarrow q$ is well-typed in $P$ in case $P \cup\left\{n \cdot x_{1} \ldots x_{k} \longrightarrow q\right\}$ is well-typed.

The second and third premise in the (Cons) rule say that the types of $n \cdot x_{1} \ldots \ldots x_{k}$ and $q$ should be both $\perp$. This guarantees that we can only rewrite terms of type $\perp$.

Example 3.8 1. Recall the term Zero with rule Zero. $c_{1} \cdot c_{2} \longrightarrow c_{1}$. We easily verify that this rule is well-typed if we let Zero $: \perp \rightarrow($ Nat $\rightarrow \perp) \rightarrow \perp$, i.e. Zero : Nat.

2. Similarly, recall the rule for Succ: Succ.x. $c_{1} \cdot c_{2} \longrightarrow c_{2} \cdot x$. It is well-typed if we let Succ : Nat $\rightarrow$ $\perp \rightarrow($ Nat $\rightarrow \perp) \rightarrow \perp$, i.e. Succ : Nat $\rightarrow$ Nat. 
3. Recalling the definition of AddCBV in Example 1.1 we see that AddCBV takes arguments of type Nat, Nat and Nat $\rightarrow \perp$ to produce a term of type $\perp$. So the rule is well-typed if we take AddCBV : Nat $\rightarrow$ Nat $\rightarrow \neg \neg$ Nat. $\left(N B\right.$. AddCBV ${ }^{\prime}:$ Nat $\rightarrow(\neg$ Nat $) \rightarrow$ Nat $\rightarrow \perp$.)

4. For the definition of AddCBN in Example 2.1] we see that AddCBN : Nat $\rightarrow$ Nat $\rightarrow$ Nat.

5. To type the rule for Omega in Example 2.5 we need Omega : $\sigma \rightarrow \perp$ with $\sigma=\sigma \rightarrow \perp$. But there is no type $\sigma$ for which $\sigma=\sigma \rightarrow \perp$, so the rule for Omega is not well-typed.

We have the following properties.

Lemma 3.9 1. [Substitution] If $n: \tau \in \Sigma, \vdash_{\Sigma} t: \sigma$ and $\vdash_{\Sigma} q: \tau$, then $\vdash_{\Sigma} t[q / n]: \sigma$.

2. [Subject reduction] If $\vdash_{\Sigma} t: \sigma$, and $t \longrightarrow p$, then $\sigma=\perp$ and $\vdash_{\Sigma} p: \perp$.

Proof 1. By induction on the derivation of $\vdash_{\Sigma} t: \sigma$.

2. Using the first property. If $t \longrightarrow p$ by the rule $n \cdot x_{1} \ldots . x_{k} \longrightarrow q$, then $t=n \cdot t_{1} \ldots . t_{k}$ and $p=$ $q\left[t_{1} / x_{1}, \ldots, t_{k} / x_{k}\right]$.

We have $x_{1}: A_{1}, \ldots, x_{k}: A_{k} \vdash_{\Sigma} q: \perp$ and $x_{1}: A_{1}, \ldots, x_{k}: A_{k} \vdash_{\Sigma} n \cdot x_{1} \ldots \ldots x_{k}: \perp$, so by substitution (where we now substitute terms for variables, but the argument is the same) we have $t: \perp$ and $p: \perp$.

We have the following Corollary of the above and of Corollary 2.4

Corollary 3.10 If $M$ and $N$ are well-typed terms of type $\perp$ and $M=_{P} N$, then $M \approx_{P} N$.

\subsection{Data types in $\mathrm{CC}$}

We have seen the definitions of the types of booleans, natural numbers and lists in Example 3.3. Here we give a general way of defining constructors and first order algebraic data types in CC. (That is, for now we don't allow higher order types in the constructor types.)

Definition 3.11 A first order data type will be written as

$$
\begin{aligned}
& \text { data-type } D \quad \text { with constructors } \\
& \mathbf{C}_{1}^{D} \quad: \quad D_{1}^{1} \rightarrow \ldots \rightarrow D_{a_{1}}^{1} \rightarrow D \\
& \\
& \qquad \mathbf{C}_{n}^{D} \quad \ldots \quad D_{1}^{n} \rightarrow \ldots \rightarrow D_{a_{n}}^{n} \rightarrow D
\end{aligned}
$$

where each of the $D_{j}^{i}$ is either $D$ or a type expression that does not contain $D$. If $D$ is clear from the context, we will omit it as a superscript and write $\mathbf{C}_{i}$ instead of $\mathbf{C}_{i}^{D}$.

This defines an algebraic data-type $D$ with $n$ constructors with names $\mathbf{C}_{1}, \ldots, \mathbf{C}_{n}$. Each constructor $\mathbf{C}_{i}$ has arity $a_{i}$, which can also be 0 , and then the constructor is a constant.

Convention 3.12 To simplify notation later, we abbreviate the list of argument types of a constructor, writing $D^{1}$ for $D_{1}^{1} \ldots D_{a_{1}}^{1}$ etc, in a style similar to uncurrying.

For every constructor we will introduce a name in $\mathrm{CC}$ and a rule that defines it. This program rule acts as a destructor of $D$. If a term of type $D$ has constructor $\mathbf{C o n s}_{i}^{D}$ as its head, all the arguments of that constructor $t_{1}^{i}, \ldots, t_{a_{i}}^{i}$ will be returned to the corresponding continuation $c_{i}$. 
Example 3.13 Consider the algebraic data type of lists over a type A, $\mathbf{L i s t}_{A}$ : In Example 3.3 we have defined this data-type in $C C \mu T . \perp \rightarrow(A \rightarrow T \rightarrow \perp) \rightarrow \perp$. The constructors for lists are added to CC by introducing the following program rules to our program, where Nil : $\perp$ and Cons : $A \rightarrow$ List $_{A} \rightarrow \perp$.

$$
\begin{aligned}
\text { Nil.c } c_{1} \cdot c_{2} & \longrightarrow c_{1} \\
\text { Cons. } x_{1} \cdot x_{2} \cdot c_{1} \cdot c_{2} & \longrightarrow c_{2} \cdot x_{1} \cdot x_{2}
\end{aligned}
$$

We now give the general definition of first order data-type in CC.

Definition 3.14 Given a first order data type $D$ as in Definition 3.11 with $n$ constructors, where, for $1 \leq i \leq n, \mathbf{C}_{i}: D_{1}^{i} \rightarrow \ldots D_{a_{i}}^{i} \rightarrow D$, we define the following type $D$ in $C C$.

$$
D:=\mu X .\left(D^{1}[X / D] \rightarrow \perp\right) \rightarrow \ldots \rightarrow\left(D^{n}[X / D] \rightarrow \perp\right) \rightarrow \perp .
$$

For $i \in[1 \ldots n]$, we add the following constructor $\operatorname{Cons}_{i}^{D}$ to the signature $\Sigma$.

$$
\operatorname{Cons}_{i}^{D}: D^{i} \rightarrow D
$$

Finally, we add for each $i(1 \leq i \leq n)$ the following program rule that acts as a destructor for $D$.

$$
\operatorname{Cons}_{i}^{D} \cdot x_{1}^{i} \ldots x_{a_{i}}^{i} \cdot c_{1} \ldots c_{n} \longrightarrow c_{i} \cdot x_{1}^{i} \ldots \ldots x_{a_{i}}^{i}
$$

So, in CC we always have $\operatorname{arity}\left(\mathbf{C o n s}_{i}^{D}\right)=a_{i}+n$. Example 3.13 conforms with this definition. The constructors are well-typed in $\mathrm{CC}$ because we have the equation

$$
D=\left(D^{1} \rightarrow \perp\right) \rightarrow \ldots \rightarrow\left(D^{n} \rightarrow \perp\right) \rightarrow \perp
$$

Notation 3.15 Let $D$ be a data type and $\mathbf{D}$ its representation as a type in Continuation Calculus. If $d: D$ (so $d$ is a data type element of $D$ ), we denote by $\langle d\rangle$ : $\mathbf{D}$ the encoding of $d$ as a term in CC. (So $\langle d\rangle$ is defined in the canonical way using the constructors of Definition 3.14)

Convention 3.16 Unless otherwise specified, $D_{j}^{i}$ is the type of the $j^{\text {th }}$ argument of the $i^{\text {th }}$ constructor of data type $D$. In the case of $\mathbf{L i s t}_{A}: D_{1}^{2}=A, D_{2}^{2}=\mathbf{L i s t}_{A}$.

We often give the typing of terms via a derivation rule.

$$
\frac{x_{1}: D_{1}^{i} \ldots x_{a_{i}}: D_{a_{i}}^{i} \quad c_{1}: D^{1} \rightarrow \perp \ldots c_{n}: D^{n} \rightarrow \perp}{\operatorname{Cons}_{i}^{D} \cdot x_{1} \ldots x_{a_{i}} \cdot c_{1} \ldots c_{n}: \perp}
$$

\subsection{Iteration schemes}

In this section we give iteration schemes for continuation calculus that provides general mechanisms for defining functions by recursion. An iteration scheme defines recursive functions in a general way, ensuring well-definedness and termination for these functions. In CC we have a call-by-name and a call-by-value variant of the iteration scheme. 
The simplest and most well-know form of iteration is over $\mathbb{N}$ : Given $b: B, f: B \rightarrow B$, the function $\mathbf{I t}(b, f): \mathbb{N} \rightarrow B$ defined by iteration from $b$ and $f$, is given by

$$
\mathbf{I t}(b, f)(n)= \begin{cases}b & \text { if } n=0 \\ f(\mathbf{I t}(b, f)(m)) & \text { if } n=m+1\end{cases}
$$

An iterator $\mathbf{I t}_{D}$ for a general data-type $D$ (following the general scheme for first order data-types in Definition 3.11) to some type $B$ has the following type:

$$
\frac{f_{1}: D^{1}[B / D] \rightarrow B \quad \ldots \quad f_{n}: D^{n}[B / D] \rightarrow B}{\mathbf{I t}_{D} f_{1} \ldots f_{n}: D \rightarrow B}
$$

with

$$
\mathbf{I t}_{D} f_{1} \ldots f_{n}\left(\mathbf{C}_{i} v_{1} \ldots v_{a_{i}}\right)=f_{i} V_{1} \ldots V_{a_{i}}
$$

where $V_{j}=\mathbf{I t}_{D} f_{1} \ldots f_{n} v_{j}$ if $v_{j}: D$ and $V_{j}=v_{j}$ otherwise.

This is not yet the correct type for an iteration scheme in CC. We do not yet have any continuations as parameters. We will provide separate $\mathrm{CBN}$ and $\mathrm{CBV}$ iteration schemes below.

\subsection{Call-by-name iterators}

For a call-by-name iterator for a data-type $D$, we also have to consider the return data-type $B$. CBV calculates the entire return value, but for $\mathrm{CBN}$ it is enough to return the proper continuations with the proper parameters after calculating only one step in the recursion. So the $\mathrm{CBN}$-iterator also passes around the continuations to the resulting values. If result type $B$ has $m$ constructors, then the iterator ItCBN $\mathbf{N}_{D \rightarrow B}$ also needs $m$ continuations as arguments. This differs from a call-by-value iterator, where we only have one continuation.

Let in the following, $D$ be a data-type with $n$ constructors $\left(\mathbf{C}_{1}^{D}, \ldots, \mathbf{C}_{n}^{D}\right)$ and $B$ a data-type with $m$ constructors.

Definition 3.17 We define the call-by-name iterator for type $D$ to type $B$ as follows. We first give the types of the new names. We abbreviate $f_{1} \ldots f_{n}$ to $\vec{f}, c_{1} \ldots c_{m}$ to $\vec{c}$ and $x_{1} \ldots x_{a_{i}}$ to $\vec{x}$.

$$
\begin{gathered}
\frac{f_{1}: D^{1}[B / D] \rightarrow B \ldots f_{n}: D^{n}[B / D] \rightarrow B \quad c_{1}: B^{1} \rightarrow \perp \ldots c_{m}: B^{m} \rightarrow \perp \quad x: D}{\mathbf{I t C B N}_{D \rightarrow B} \cdot \vec{f} \cdot x \cdot \vec{c}: \perp} \\
\frac{f_{1}: D^{1}[B / D] \rightarrow B \ldots f_{n}: D^{n}[B / D] \rightarrow B \quad c_{1}: B^{1} \rightarrow \perp \ldots c_{m}: B^{m} \rightarrow \perp \quad x_{1}: D_{1}^{i} \ldots x_{a_{i}}^{i}: D_{a_{i}}^{i}}{\mathbf{I t C B N}_{D \rightarrow B}^{i} \cdot \vec{f} \cdot \vec{c} \cdot x_{1} \ldots x_{a_{i}}: \perp}
\end{gathered}
$$

The program rules are

$$
\operatorname{ItCBN}_{D \rightarrow B} \cdot \vec{f} \cdot x \cdot \vec{c} \longrightarrow x \cdot\left(\operatorname{ItCBN}_{D \rightarrow B}^{1} \cdot \vec{f} \cdot \vec{c}\right) \ldots\left(\operatorname{ItCBN}_{D \rightarrow B}^{\mathrm{n}} \cdot \vec{f} \cdot \vec{c}\right),
$$

and for $i \in[1 \ldots n]$ :

$$
\begin{gathered}
\mathbf{I t C B N} \mathbf{N}_{D \rightarrow B}^{\mathrm{i}} \cdot \vec{f} \cdot \vec{c} \cdot \vec{x} \longrightarrow f_{i} \cdot b\left(x_{1}\right) \ldots . b b\left(x_{a_{i}}\right) \cdot \vec{c} \\
\text { with } b(x)= \begin{cases}\mathbf{I t C B N}_{D \rightarrow B} \cdot \vec{f} \cdot x & \text { if } x: D \\
x & \text { otherwise }\end{cases}
\end{gathered}
$$


In Section 4, we give in Example 4.1 the call-by-name iterator for $\mathbf{L i s t}_{A}$ to Nat. The following can easily be checked. (See Definition 3.7 for the formal definition of well-typed rules.)

Lemma 3.18 The rules given in Definition 3.17 are well-typed.

\subsection{Call-by-value iterators}

Call-by-value iterators differ from their call-by-name cousins in the sense that the result of the computation is 'normalized' or fully evaluated at the end of the computation.

Definition 3.19 We define the call-by-value iterator for a type $D$ to $B$ as follows. (We abbreviate $f_{1} \ldots f_{n}$ to $\vec{f}$.)

$$
\frac{f_{1}: D^{1}[B / D] \rightarrow \neg \neg B \ldots f_{n}: D^{n}[B / D] \rightarrow \neg \neg B \quad c: \neg B \quad d: D}{\operatorname{ItCBV}_{D \rightarrow B} \vec{f} c d: \perp}
$$

and for $i \in[1, n]$ and $j \in\left[1, a_{i}\right]$, under the same typing hypotheses for $\vec{f}$ and $c$ :

$$
\frac{x_{j}: D_{j}^{i} \ldots x_{a_{i}}: D_{a_{i}}^{i} \quad r_{1}: D_{1}^{i}[B / D] \ldots r_{j-1}: D_{j_{1}}^{i}[B / D]}{\mathbf{I t C B V}_{D \rightarrow B}^{\mathrm{i}, j} \cdot \vec{f} \cdot c \cdot x_{j} \ldots x_{a_{i}} \cdot r_{1} \ldots r_{j-1}: \perp}
$$

The program rules are

$$
\operatorname{ItCBV}_{D \rightarrow B} \cdot \vec{f} \cdot c \cdot x \longrightarrow x \cdot\left(\operatorname{ItCBV}_{D \rightarrow B}^{1,1} \cdot \vec{f} . c\right) \ldots .\left(\operatorname{ItCBV}_{D \rightarrow B}^{\mathrm{n}, 1} \cdot \vec{f} . c\right)
$$

and for $i \in[1, n]$ and $j \in\left[1, a_{i}\right]$ :

$$
\begin{aligned}
& \mathbf{I t C B V}_{\vec{D} \rightarrow B}^{\mathrm{i}, j} \cdot \vec{f} \cdot c \cdot x_{j} \ldots x_{a_{i}} \cdot r_{1} \ldots r_{j-1} \longrightarrow L H S \\
& L H S= \begin{cases}\mathbf{I t C B V}_{D \rightarrow B} \cdot \vec{f} \cdot\left(\mathbf{I t C B V} \mathbf{V}_{D \rightarrow B}^{\mathrm{i}, j+1} \cdot \vec{f} \cdot c \cdot x_{j+1} \ldots x_{a_{i}} \cdot r_{1} \ldots r_{j-1}\right) \cdot x_{j} & \text { if } x_{j}: D \\
\mathbf{I t C B V}_{D \rightarrow B}^{\mathrm{i}, j+1} \cdot \vec{f} \cdot c \cdot x_{j+1} \ldots x_{a_{i}} \cdot r_{1} \ldots r_{j-1} \cdot x_{j} & \text { otherwise }\end{cases} \\
& \mathbf{I t C B V}_{D \rightarrow B}^{\mathrm{i}, a_{i}+1} \cdot \vec{f} \cdot c \cdot r_{1} \ldots r_{a_{i}} \quad \longrightarrow \quad f_{i} \cdot r_{1} \ldots r_{a_{i}} \cdot c
\end{aligned}
$$

The technical subtlety in the call-by-value reduction rule lies in the fact that, in case data-type $D$ has a constructor with more than one recursive sub-term (e.g. in the case of binary trees, where we have 'join', taking two sub-trees), we have to evaluate all recursive sub-terms. The reduction rule makes sure that we do that and reduce to a complete value before calling the function. The following lemma helps in better understanding the terms $\mathbf{I t C B V} \mathbf{i}_{D \rightarrow B}^{\mathrm{i}, j} . \vec{f} . c$. in Definition 3.19 .

Lemma 3.20 For $j \in\left[1 \ldots a_{i}\right]$, given $x_{j}: D_{j}^{i} \ldots x_{a_{i}}: D_{a_{i}}^{i}$ and $r_{1}: D_{1}^{i}[B / D] \ldots r_{j-1}: D_{j_{1}}^{i}[B / D]$, the reduct of ItCBV $\mathrm{I}_{D \rightarrow B}^{\mathrm{i}, j} \cdot \vec{f} . c \cdot x_{j} \ldots x_{a_{i}} \cdot r_{1} \ldots r_{j-1}$ is of type $\perp$.

Proof For $j=a_{i}$, the result is immediate, for other $j$, the result follows from the result for $j+1$, making a case distinction between $D_{j}^{i}[B / D]=D_{j}^{i}$ or $D_{j}^{i}[B / D]=B$.

The following now easily follows.

Lemma 3.21 The rules given in Definition 3.19 are well-typed. 
Example 3.22 For the iterator from Nat to Nat, this amounts to the following

$$
\begin{aligned}
& \operatorname{ItCBV}_{\mathbf{N a t} \rightarrow \mathbf{N a t}} \cdot f_{1} \cdot f_{2} \cdot c \cdot x \longrightarrow x \cdot\left(\mathbf{I t C B V}_{\mathbf{N a t} \rightarrow \mathbf{N a t}}^{\text {Zero }} \cdot f_{1} \cdot f_{2} \cdot c\right) \cdot\left(\mathbf{I t C B V}_{\mathbf{N a t} \rightarrow \mathbf{N a t}}^{\text {Succ, }} \cdot f_{1} \cdot f_{2} \cdot c\right) \\
& \quad \operatorname{ItCBV}_{\mathbf{N a t} \rightarrow \mathbf{N a t}}^{\text {Zero,1 }} \cdot f_{1} \cdot f_{2} \cdot c \longrightarrow f_{1} \cdot c \\
& \operatorname{ItCBV}_{\mathbf{N a t} \rightarrow \mathbf{N a t}}^{\text {Succ, }} \cdot f_{1} \cdot f_{2} \cdot c \cdot x_{1} \longrightarrow \mathbf{I t C B V}_{\mathbf{N a t} \rightarrow \mathbf{N a t}} \cdot f_{1} \cdot f_{2} \cdot\left(\mathbf{I t C B V}_{\mathbf{N a t} \rightarrow \mathbf{N a t}}^{\text {Succ, }} \cdot f_{1} \cdot f_{2} \cdot c\right) \cdot x_{1} \\
& \operatorname{ItCBV}_{\mathbf{N a t} \rightarrow \mathbf{N a t}}^{\text {Succ, }} \cdot f_{1} \cdot f_{2} \cdot c \cdot r_{1} \longrightarrow f_{2} \cdot r_{1} \cdot c
\end{aligned}
$$

This can be compressed a bit if we replace $\mathbf{I t C B V} \mathbf{V}_{\mathbf{N a t} \rightarrow \mathbf{N a t}}^{\mathrm{Zero}, 1} \cdot f_{1} . f_{2} . c$ by $f_{1} . c$.

Another simplification that we can do is to replace some auxiliary names that are introduced in the iteration scheme by a $\lambda$-term. For example we can replace $\mathbf{I t C B V}_{\mathbf{N a t} \rightarrow \mathbf{N a t}}^{\text {Suc }, 2}$ by the new 'name' $\left(\lambda \mathrm{f}_{1}, \mathrm{f}_{2}, \mathrm{c}, \mathrm{r}_{1} \mapsto\right.$ $\left.\mathrm{f}_{2} \cdot \mathrm{r}_{1} . \mathrm{c}\right)$. The convention for such a name is that

$$
\left(\lambda \mathrm{f}_{1}, \mathrm{f}_{2}, \mathrm{c}, \mathrm{r}_{1} \mapsto \mathrm{f}_{2} \cdot \mathrm{r}_{1} \cdot \mathrm{c}\right) \cdot f_{1} \cdot f_{2} \cdot c \cdot r_{1} \longrightarrow f_{2} \cdot r_{1} \cdot c .
$$

So, the arity of the new name is the number of arguments of the $\lambda$ and its program rule is given by the body. Now the rules for $\mathbf{I t C B V _ { \mathbf { N a t } } \rightarrow \text { Nat }}$ simplify to

$$
\begin{aligned}
& \operatorname{ItCBV}_{\mathbf{N a t} \rightarrow \mathbf{N a t}} \cdot f_{1} \cdot f_{2} \cdot c \cdot x \longrightarrow x \cdot\left(f_{1} \cdot c\right) \cdot\left(\mathbf{I t C B V}_{\mathbf{N a t} \rightarrow \mathbf{N a t}}^{\text {Succ, }} \cdot f_{1} \cdot f_{2} \cdot c\right) \\
& \operatorname{ItCBV}_{\mathbf{N a t} \rightarrow \mathbf{N a t}}^{\text {Succ, }} \cdot f_{1} \cdot f_{2} \cdot c \cdot x_{1} \longrightarrow \mathbf{I t C B V}_{\mathbf{N a t} \rightarrow \mathbf{N a t}} \cdot f_{1} \cdot f_{2} \cdot\left(\left(\lambda f_{1}, f_{2}, c, r_{1} \mapsto f_{2} \cdot r_{1} \cdot c\right) \cdot f_{1} \cdot f_{2} \cdot c\right) \cdot x_{1}
\end{aligned}
$$

In Section 4, we show more examples, notably in Example 4.2 we give the call-by-value iterator for List A $_{A}$ and we show how to program the 'length' function with it.

\subsection{Rules for programming with data types in $\mathrm{CC}$}

Starting from the constructors for first order data types and the call-by-name and call-by-value iterators we can program new functions from existing ones. However, due to the fact that we are in CC and not in $\lambda$-calculus, we need some additional 'glue' to make flexible use of the iteration scheme to define functions.

Example 3.23 Given ItCBV $\mathbf{N a t}_{\rightarrow \mathrm{Nat}}$ we can define $\mathbf{A d d C B V}$ as follows.

$$
\operatorname{AddCBV} . m . n . c:=\operatorname{ItCBV}_{\mathbf{N a t} \rightarrow \mathbf{N a t}} \cdot\left(F_{1} \cdot m\right) \cdot F_{2} \cdot c \cdot n
$$

where $F_{1}$ and $F_{2}$ are defined by

$$
\begin{aligned}
& F_{1} . x . c \longrightarrow c . x \\
& F_{2} . x . c \longrightarrow c .(\text { Succ } . x)
\end{aligned}
$$

So, we need 2 auxiliary functions to define $\mathbf{A d d C B V}$ in terms of $\mathbf{I t C B V} \mathbf{V}_{\mathbf{N a t} \rightarrow \mathbf{N a t}}$. In terms of Example 3.23 we need the names $(\lambda \mathrm{x}, \mathrm{c} \mapsto \mathrm{c} . \mathrm{x})$, which is $F_{1}$ and $(\lambda \mathrm{x}, \mathrm{c} \mapsto \mathrm{c}$. (Succ.x $\left.)\right)$, which is $F_{2}$.

The example shows that, to really profit from the expressivity of the iteration schemes, we must allow the addition of 'simple' functions. These are functions that have a non-circular definition. 
Definition 3.24 A non-circular program rule is a rule of the form

$$
n \cdot x_{1} \ldots x_{k} \longrightarrow q,
$$

where the names occurring in $q$ are restricted to the constructors (Definition 3.14) and the iterators (CBN, Definition 3.17 and CBV, Definition 3.19).

We define $\mathbb{P}$ as the set of program rules that contains constructors for all data types (Definition 3.14), iterators for all data types (CBN, Definition 3.17 and CBV, 3.19) and arbitrarily many non-circular rules.

So, $\mathbb{P}$ is an "open set": it contains constructors and iterators for all (infinitely many) data-types that we can define, and it includes arbitrarily many "non-circular rules" that can be added when desired. This is needed to really define functions using the iteration schemes.

\subsection{Translating between call-by-name and call-by-value}

We can mediate between the call-by-name and the call-by-value representations of data by defining a function StoreNat : Nat $\rightarrow \neg \neg$ Nat and a function UnstoreNat : $\neg \neg$ Nat $\rightarrow$ Nat. Recall from Notation 3.15 that $\langle n\rangle$ is defined as $\mathbf{S u c c}^{n}$.Zero. The function StoreNat acts as a storage operator in the sense of Krivine [6] in the sense that for $t:$ Nat with $t \approx\langle n\rangle$ and $c:$ Nat $\rightarrow \perp$,

$$
\text { StoreNat.t.c } \longrightarrow c .\langle n\rangle \text {. }
$$

So, StoreNat first evaluates the argument $t$ of type Nat completely before passing it on to the continuation c. The term StoreNat.t.c can be defined as AddCBV.t.Zero.c, but we can also define it directly by

$$
\begin{aligned}
& \text { StoreNat. } n . r \quad \longrightarrow \quad n .(r . \text { Zero).(StoreNatA. } r) \\
& \text { StoreNatA.r.m } \longrightarrow \text { StoreNat.m.(StoreNatB.r) } \\
& \text { StoreNatB } . r . m^{\prime} \longrightarrow r .\left(\text { Succ } . m^{\prime}\right)
\end{aligned}
$$

It is easy to verify that StoreNat : Nat $\rightarrow \neg \neg$ Nat. (Note that StoreNatA, StoreNatB : $\neg$ Nat $\rightarrow \neg$ Nat.)

In the reverse direction, we have UnstoreNat : $\neg \neg$ Nat $\rightarrow$ Nat, defined by, given $f: \neg \neg$ Nat,

$$
\begin{aligned}
& \text { UnstoreNat.f.z.s } \longrightarrow f \text {.(UseNat.z.s) } \\
& \text { UseNat.z.s.n } \longrightarrow \text { n.z.s }
\end{aligned}
$$

Then UnstoreNat : $\neg \neg$ Nat $\rightarrow$ Nat. (Note that UseNat $: \perp \rightarrow \neg$ Nat $\rightarrow \neg$ Nat.)

Lemma 3.25 For all $t:$ Nat and $n \in \mathbb{N}$ with $t \approx\langle n\rangle$, StoreNat.t.c $\longrightarrow c$. $\langle n\rangle$. For all $n \in \mathbb{N}$, UnstoreNat.(StoreNat. $\langle n\rangle) \approx\langle n\rangle$.

Proof For the first, we note that, if $t \approx\langle n\rangle$, then: (i) in case $n=0, t . z . s \longrightarrow z$; (ii) in case $n=$ $m+1$, t.z.s $\longrightarrow s . q$ for some $q$ with $q \approx\langle m\rangle$. Then we prove the following by induction on $n$ and $p$ : StoreNat. $\langle n\rangle .\left(\right.$ StoreNatB $\left.^{p} . r\right) \longrightarrow r$. $\left(\right.$ Succ $^{n+p}$.Zero $)$.

For the second, we prove UnstoreNat.(StoreNat. $\langle n\rangle$ ).z.s $=_{P}\langle n\rangle . z . s$, which is sufficient by Corollary 3.10. We compute:

$$
\begin{aligned}
\text { UnstoreNat. }(\text { StoreNat. }\langle n\rangle) . z . s & \longrightarrow \text { StoreNat. }\langle n\rangle .(\text { UseNat.z.s) } \\
& \longrightarrow \text { UseNat.z.s. }\langle n\rangle \\
& \longrightarrow\langle n\rangle \cdot z . s
\end{aligned}
$$


Thus, UnstoreNat.(StoreNat. $\langle n\rangle) . z . s={ }_{P}\langle n\rangle . z . s$ which was what we had to prove.

The map StoreNat can be seen as adding a double negation, whereas UnstoreNat can be seen as a classical double negation law, UnstoreNat : $\neg \neg$ Nat $\rightarrow$ Nat. Note that the fact that $\neg \neg$ Nat $\rightarrow$ Nat is inhabited is not a surprise, because Nat is a negative type (ending in $\rightarrow \perp$ ). The precise connection with classical logic remains to be studied.

The storage and 'unstorage' operators can most likely also be defined for other data types.

More interesting to study further is the fact that we can combine call-by-name and call-by-value functions. We detail this for natural numbers.

Example 3.26 If we have $f_{1}:$ Nat and $f_{2}:$ Nat $\rightarrow$ Nat, $c_{1}: \perp, c_{2}:$ Nat $\rightarrow \perp$ and $n:$ Nat, then

$$
\operatorname{ItCBN}_{\mathbf{N a t} \rightarrow \mathbf{N a t}} \cdot f_{1} \cdot f_{2} \cdot n \cdot c_{1} \cdot c_{2}: \perp
$$

gives a call-by-name iteration. However, one can also first define $\hat{f}_{1}: \neg \neg \mathbf{N a t}, \hat{f}_{2}:$ Nat $\rightarrow \neg \neg$ Nat and $\widehat{\left(c_{1}, c_{2}\right)}: \mathbf{N a t} \rightarrow \perp$ by

$$
\begin{aligned}
\hat{f}_{1} \cdot c & \longrightarrow c \cdot f_{1} \\
\hat{f}_{2} \cdot n \cdot c & \longrightarrow c \cdot\left(f_{2} \cdot n\right) \\
\left(c_{1}, c_{2}\right) \cdot n & \longrightarrow n \cdot c_{1} \cdot c_{2}
\end{aligned}
$$

Then, for $n$ : Nat, we have

$$
\operatorname{ItCBV}_{\mathrm{Nat} \rightarrow \mathrm{Nat}} \cdot \hat{f}_{1} \cdot \hat{f_{2}} \cdot \widehat{\left(c_{1}, c_{2}\right)} \cdot n: \perp
$$

which gives call-by-value iteration. So, using this transformation (from $f_{1}$ to $\hat{f}_{1}$ etc.) one can use the call-by-name functions to compute call-by-value.

\section{Examples of iterators and programs}

Example 4.1 This is the call-by-name iterator for List $_{A}$ to Nat:

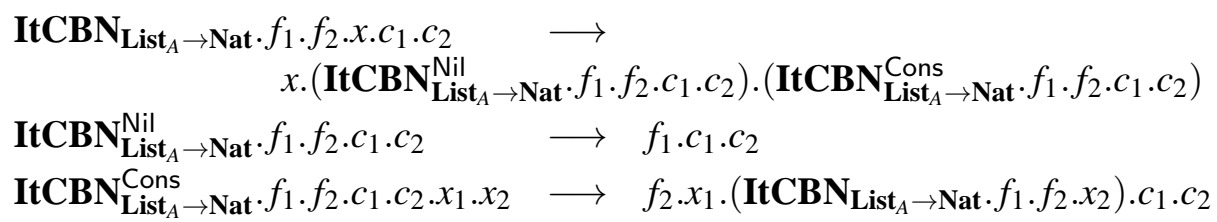

Example 4.2 The scheme of Definition 3.19 yields the following call-by-value iterator for $\mathbf{L i s t}_{A}$ to B:

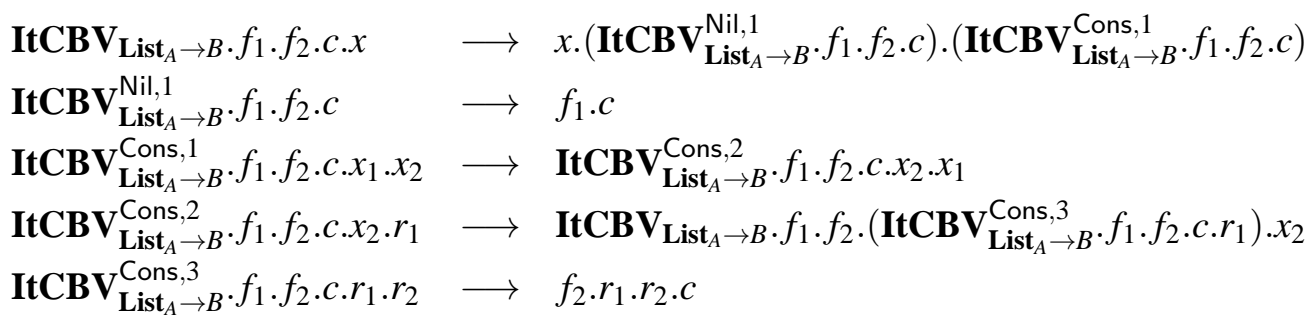


We note that the number of program rules we need is highly dependent on the arity of the constructors Nil and Cons. Since Nil has no parameters, one rule is enough to define the operation on Nil. Cons on the other hand has two parameters. Because of this we get three program rules: one for evaluating each parameter of the constructor and one general rule that redirects every parameter to the corresponding program rule.

We now show the use of the iterators by providing the implementation of the function Length. We use the iterators for $\mathbf{L i s t}_{A}$ from Example 4.1 and Example 4.2.
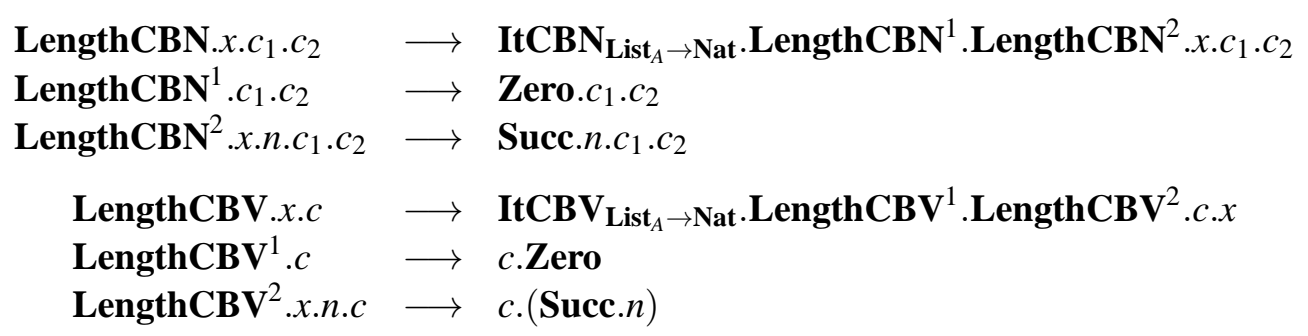

We prove for $\mathbb{N}$ that the two iterator schemes (CBN and CBV) indeed compute the desired results. We expect that this proof can easily be extended to prove the semantics of our iteration schemes for any first-order algebraic data-type. We leave this for future work.

We assume $D$ to be a data type which has a representation in Continuation Calculus, $\mathbf{D}$, with a representation such that $\langle d\rangle: \mathbf{D}$, for $d: D$. We now define what it means that a function over a data-type is represented in CC.

Definition 4.3 We say that $f_{1}: \neg \neg \mathbf{D}$ CBV-represents $d: D$ and $f_{2}: \mathbf{D} \rightarrow \neg \neg \mathbf{D}$ CBV-represents $F: D \rightarrow D$, if for all $c: \mathbf{D} \rightarrow \perp$ and $n \in \mathbb{N}$ we have

$$
\begin{gathered}
f_{1} . c \longrightarrow c \cdot\langle d\rangle \\
f_{2} \cdot\langle n\rangle . c \longrightarrow c \cdot\langle F(n)\rangle
\end{gathered}
$$

The following Theorem states the semantic correctness of $\mathbf{I t C B V}_{\mathbf{N a t} \rightarrow D}$ in CC. The proof can be found in Section 6 of the Appendix.

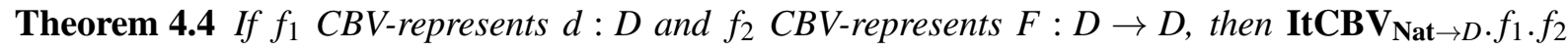
$C B V$-represents $\mathbf{I t}(d, F)$, that is: for all $c: \mathbf{D} \rightarrow \perp$ and all $n \in \mathbb{N}$ we have

$$
\operatorname{ItCBV}_{\mathbf{N a t} \rightarrow D} \cdot f_{1} \cdot f_{2} \cdot c .\langle n\rangle \longrightarrow c .\langle(\mathbf{I t}(d, F))(n)\rangle
$$

Definition 4.5 If $D$ has $m$ constructors, we say that $f_{1}: \mathbf{D}$ CBN-represents $d: D$ and $f_{2}: \mathbf{D} \rightarrow \mathbf{D}$ CBNrepresents $F: D \rightarrow D$, if for all $c_{i}: \mathbf{D}^{i}$ and $n \in \mathbb{N}$ we have (writing $\vec{c}=c_{1} \ldots c_{m}$ ):

$$
\begin{gathered}
f_{1} \cdot \vec{c} \approx\langle d\rangle \cdot \vec{c} \\
f_{2} \cdot\langle n\rangle . \vec{c} \approx\langle F(n)\rangle \cdot \vec{c}
\end{gathered}
$$

The following Theorem states the semantic correctness of $\mathbf{I t C B N} \mathbf{N a t}_{\mathbf{N a D}}$ in CC. The proof can be found in Section 6 of the Appendix.

Theorem 4.6 If $D$ has $m$ constructors, $f_{1}$ CBN-represents $d: D$ and $f_{2}$ CBN-represents $F: D \rightarrow D$, then $\mathbf{I t C B N}_{\mathbf{N a t} \rightarrow D} \cdot f_{1} \cdot f_{2} C B N$-represents $\mathbf{I t}(d, F)$. That is, for all $c_{i}: \mathbf{D}^{i}$ and all $n \in \mathbb{N}$ :

$$
\mathbf{I t C B N}_{\mathbf{N a t} \rightarrow D} \cdot f_{1} \cdot f_{2} \cdot\langle n\rangle \cdot \vec{c} \approx\langle(\mathbf{I t}(d, F))(n)\rangle \cdot \vec{c}
$$




\section{Future Work and Conclusions}

As future work, we want to better understand the relation with classical logic, which we have suggested in Section 4. Here we have also defined storage (and unstorage) operators, which we would like to define in general for all data types. The possibility to combine call-by-value and call-by-name in a flexible way, which is directed by the types, is an interesting feature, which warrants further study. The fact that computation is completely deterministic and that the function definition of $f$ itself determines whether $f$ is cbv or cbn, makes this combining of cbv and cbn very perspicuous.

The continuations in this paper are limited, and do not include delimited continuations. (More examples using continuations can be found in [2].) It would be interesting to see if delimited continuations can be added.

In a forthcoming paper [4] we prove the termination of all $\mathrm{CC}$ terms written using the program rules of $\mathbb{P}$. This is done by translating CC with these rules to a typed $\lambda$-calculus with (cbv and cbn) iterators. We wish to further study the precise translations and connections between $\mathrm{CC}$ and (typed) $\lambda$-calculus.

\section{References}

[1] M. Abadi, L. Cardelli \& G. Plotkin (1993): Types for the Scott Numerals. http://lucacardelli.name/ Papers/Notes/scott2.pdf.

[2] B. Geron \& H. Geuvers (2013): Continuation calculus. In: Proceedings of COS 2013, EPTCS 127, pp. 66-85, doi:10.4204/EPTCS.127.5.

[3] Bram Geron (2013): Continuation Calculus, master's thesis. http://alexandria.tue.nl/extra1/ afstversl/wsk-i/geron2013.pdf.

[4] Herman Geuvers (2014): A typed $\lambda$-calculus with CBN and CBV iterators. To appear.

[5] J.M. Jansen (2013): Programming in the $\lambda$-Calculus: From Church to Scott and Back. In: The Beauty of Functional Code, Lecture Notes in Computer Science 8106, pp. 168-180, doi:10.1007/978-3-642-38143-0.

[6] Jean-Louis Krivine (1994): Classical Logic, Storage Operators and Second-Order lambda-Calculus. Ann. Pure Appl. Logic 68(1), pp. 53-78, doi:10.1016/0168-0072(94)90047-7.

[7] A. Miquel (2009): Classical realizability with forcing and the axiom of countable choice. http://perso. ens-lyon.fr/alexandre.miquel/habilitation/forcing.pdf.

\section{Appendix}

Proof of Theorem 4.4

Proof We recall the definition of $\operatorname{ItCBV}_{\mathbf{N a t} \rightarrow B} B$ :

$$
\begin{aligned}
& \operatorname{ItCBV}_{\mathbf{N a t} \rightarrow B} \cdot f_{1} \cdot f_{2} \cdot c \cdot x \longrightarrow x .\left(\operatorname{ItCBV}_{\mathbf{N a t} \rightarrow B}^{Z e r o, 1} \cdot f_{1} \cdot f_{2} \cdot c\right) .\left(\operatorname{ItCBV}_{\mathbf{N a t} \rightarrow B}^{\text {Succ }, 1} \cdot f_{1} \cdot f_{2} \cdot c\right) \\
& \operatorname{ItCBV}_{\mathbf{N a t} \rightarrow B}^{\text {Zero, } 1} \cdot f_{1} \cdot f_{2} \cdot c \longrightarrow f_{1} \cdot c \\
& \operatorname{ItCBV}_{\mathbf{N a t} \rightarrow B}^{\text {Succ, } 1} \cdot f_{1} \cdot f_{2} \cdot \text { c. } x_{1} \longrightarrow \mathbf{I t C B V}_{\mathbf{N a t} \rightarrow B} \cdot f_{1} \cdot f_{2} \cdot\left(\mathbf{I t C B V}_{\mathbf{N a t} \rightarrow B}^{\text {Succ }, 2} \cdot f_{1} \cdot f_{2} \cdot c\right) \cdot x_{1} \\
& \mathbf{I t C B V}_{\mathbf{N a t} \rightarrow B}^{\text {Succ }, 2} \cdot f_{1} \cdot f_{2} \cdot c \cdot r_{1} \longrightarrow f_{2} \cdot r_{1} \cdot c
\end{aligned}
$$


By induction on $n$ we prove that for all $n, P(n)$ holds, with

$$
P(n):=\mathbf{I t C B V}_{\mathbf{N a t} \rightarrow B} \cdot f_{1} \cdot f_{2} \cdot c \cdot\langle n\rangle \longrightarrow c \cdot\langle(\mathbf{I t}(x, F))(n)\rangle
$$

$P(0)$ holds, because:

$$
\begin{aligned}
& \mathbf{I t C B V}_{\mathbf{N a t} \rightarrow B} \cdot f_{1} \cdot f_{2} \cdot c \cdot\langle 0\rangle \stackrel{(3 \mathrm{a})}{\longrightarrow}\langle 0\rangle .\left(\mathbf{I t C B V}_{\mathbf{N a t} \rightarrow B}^{\text {Zero } 1} \cdot f_{1} \cdot f_{2} \cdot c\right) .\left(\mathbf{I t C B V}_{\mathbf{N a t} \rightarrow B}^{\text {Succ }, 1} \cdot f_{1} \cdot f_{2} \cdot c\right) \\
& =\text { Zero. }\left(\mathbf{I t C B V}_{\mathbf{N a t} \rightarrow B}^{\text {Zero, } \rightarrow} \cdot f_{1} \cdot f_{2} \cdot c\right) .\left(\mathbf{I t C B V}_{\mathbf{N a t} \rightarrow B}^{\text {Succ, } \rightarrow} \cdot f_{1} \cdot f_{2} \cdot c\right)
\end{aligned}
$$

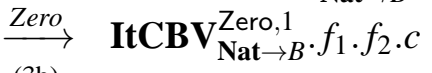

$$
\begin{aligned}
& \stackrel{(3 \mathrm{~b})}{\longrightarrow} f_{1} \cdot c \\
& \stackrel{(1)}{\longrightarrow} \quad c \cdot\langle x\rangle \\
& =c \cdot\langle(\mathbf{I t}(x, F))(0)\rangle
\end{aligned}
$$

Assume $P(n)$ holds.

$P(n+1)$ holds, because:

$$
\begin{aligned}
& \operatorname{ItCBV}_{\mathbf{N a t} \rightarrow B} \cdot f_{1} \cdot f_{2} \cdot c \cdot\langle n+1\rangle \quad \stackrel{(3 \mathrm{a})}{\longrightarrow} \quad\langle n+1\rangle .\left(\mathbf{I t C B V}_{\mathbf{N a t} \rightarrow B}^{\text {Zero }, 1} \cdot f_{1} \cdot f_{2} \cdot c\right) \cdot\left(\mathbf{I t C B ~ V}_{\mathbf{N a t} \rightarrow B}^{\text {Succ }, 1} \cdot f_{1} \cdot f_{2} \cdot c\right) \\
& =\text { Succ. }\langle n\rangle \cdot\left(\operatorname{ItCBV}_{\mathbf{N a t} \rightarrow B}^{\mathrm{Zero}, 1} \cdot f_{1} \cdot f_{2} \cdot c\right) .\left(\mathbf{I t C B V}_{\mathbf{N a t} \rightarrow B}^{\mathrm{Succ}, 1} \cdot f_{1} \cdot f_{2} \cdot c\right) \\
& \stackrel{\text { Succ }}{\longrightarrow} \operatorname{ItCBV}_{\mathbf{N a t} \rightarrow B}^{\text {Succ, } 1} \cdot f_{1} \cdot f_{2} \cdot c \cdot\langle n\rangle \\
& \stackrel{(3 \mathrm{c})}{\longrightarrow} \quad \mathbf{I t C B V}_{\mathbf{N a t} \rightarrow B} \cdot f_{1} \cdot f_{2} \cdot\left(\mathbf{I t C B V}_{\mathbf{N a t} \rightarrow B}^{\text {Succ, }} \cdot f_{1} \cdot f_{2} \cdot c\right) \cdot\langle n\rangle \\
& \stackrel{P(n)}{\longrightarrow} \quad \mathbf{I t C B V} \mathbf{V u c c}_{\mathbf{N a t} \rightarrow B}^{\text {Sur }} \cdot f_{1} \cdot f_{2} \cdot c \cdot\langle(\mathbf{I t}(x, F))(n)\rangle \\
& \stackrel{(3 \mathrm{~d})}{\longrightarrow} f_{2} \cdot\langle(\mathbf{I t}(x, F))(n)\rangle \cdot c \\
& \stackrel{(2)}{\longrightarrow} \quad c \cdot\langle F((\mathbf{I t}(x, F))(n))\rangle \\
& =c \cdot\left\langle F\left(F^{n}(x)\right)\right\rangle \\
& =\quad c \cdot\left\langle F^{n+1}(x)\right\rangle \\
& =\quad c \cdot\langle(\mathbf{I t}(x, F))(n+1)\rangle
\end{aligned}
$$

Proof of Theorem 4.6

Proof We recall the definition of $\mathbf{I t C B N} \mathbf{N}_{\mathbf{N a t} \rightarrow B}$ :

$$
\begin{aligned}
& \operatorname{ItCBN}_{\mathbf{N a t} \rightarrow B} \cdot f_{1} \cdot f_{2} \cdot x \cdot \vec{c} \longrightarrow x \cdot\left(\operatorname{ItCBN}_{\mathbf{N a t} \rightarrow B}^{\text {Zero }} \cdot f_{1} \cdot f_{2} \cdot \vec{c}\right) \cdot\left(\operatorname{ItCBN}_{\mathbf{N a t} \rightarrow B}^{\text {Succ }} \cdot f_{1} \cdot f_{2} \cdot \vec{c}\right) \\
& \operatorname{ItCBN}_{\mathbf{N a t} \rightarrow B}^{\text {Zero }} \cdot f_{1} \cdot f_{2} \cdot c \longrightarrow f_{1} \cdot \vec{c} \\
& \operatorname{ItCBN}_{\mathbf{N a t} \rightarrow B}^{\text {Succ }} \cdot f_{1} \cdot f_{2} \cdot c \cdot x_{1} \longrightarrow f_{2} \cdot\left(\operatorname{ItCBN}_{\mathbf{N a t} \rightarrow B} \cdot f_{1} \cdot f_{2} \cdot x_{1}\right) \cdot \vec{c}
\end{aligned}
$$

By induction on $n$ we prove that for all $n, P(n)$ holds, with

$$
P(n):=\mathbf{I t C B N}_{\mathbf{N a t} \rightarrow B} \cdot f_{1} \cdot f_{2} \cdot\langle n\rangle \cdot \vec{c} \approx\langle(\mathbf{I t}(x, F))(n)\rangle \cdot \vec{c}
$$


$P(0)$ holds, because:

$$
\begin{aligned}
& \operatorname{ItCBN}_{\mathbf{N a t} \rightarrow B} \cdot f_{1} \cdot f_{2} \cdot\langle 0\rangle \cdot \vec{c} \stackrel{(3 \mathrm{a})}{\longrightarrow} \quad\langle 0\rangle .\left(\operatorname{ItCBN}_{\mathbf{N a t} \rightarrow B}^{\text {Zero }} \cdot f_{1} \cdot f_{2} \cdot \vec{c}\right) .\left(\mathbf{I t C B N}_{\mathbf{N a t} \rightarrow B}^{\text {Succ }} \cdot f_{1} \cdot f_{2} \cdot \vec{c}\right) \\
& =\text { Zero. }\left(\operatorname{ItCBN} \mathbf{N}_{\mathbf{N a t} \rightarrow B}^{\text {Zero }} \cdot f_{1} \cdot f_{2} \cdot \vec{c}\right) \cdot\left(\operatorname{ItCBN} \mathbf{N a t} \rightarrow B_{\mathbf{N a c}}^{\text {Sut }} \cdot f_{1} \cdot f_{2} \cdot \vec{c}\right) \\
& \stackrel{\text { Zero }}{\longrightarrow} \text { ItCBN } \text { Nat } \rightarrow B_{\text {ero }} \cdot f_{1} \cdot f_{2} \cdot \vec{c} \\
& \stackrel{(3 \mathrm{~b})}{\longrightarrow} f_{1} \cdot \vec{c} \\
& \stackrel{(1)}{\longrightarrow}\langle x\rangle \cdot \vec{c} \\
& =\langle(\mathbf{I t}(x, F))(0)\rangle \cdot \vec{c}
\end{aligned}
$$

Assume $P(n)$ holds.

$P(n+1)$ holds, because:

$$
\begin{aligned}
& \operatorname{ItCBN}_{\mathbf{N a t} \rightarrow B} \cdot f_{1} \cdot f_{2} \cdot\langle n+1\rangle \cdot \vec{c} \quad \stackrel{(3 \mathrm{a})}{\longrightarrow} \quad\langle n+1\rangle \cdot\left(\mathbf{I t C B N}_{\mathbf{N a t} \rightarrow B}^{\text {Zero }} \cdot f_{1} \cdot f_{2} \cdot \vec{c}\right) \cdot\left(\mathbf{I t C B N}_{\mathbf{N a t} \rightarrow B}^{\text {Succ }} \cdot f_{1} \cdot f_{2} \cdot \vec{c}\right) \\
& =\quad \text { Succ. }\langle n\rangle \cdot\left(\operatorname{ItCBN}_{\mathbf{N a t} \rightarrow B}^{\text {Zero }} \cdot f_{1} \cdot f_{2} \cdot \vec{c}\right) \cdot\left(\operatorname{ItCBN}_{\mathbf{N a t} \rightarrow B}^{\text {Succ }} \cdot f_{1} \cdot f_{2} \cdot \vec{c}\right) \\
& \stackrel{\text { Succ }}{\longrightarrow} \quad \operatorname{ItCBN}_{\mathbf{N a t} \rightarrow B}^{\text {Succ }} \cdot f_{1} \cdot f_{2} \cdot \vec{c} \cdot\langle n\rangle \\
& \stackrel{(3 \mathrm{c})}{\longrightarrow} \quad f_{2} \cdot\left(\mathbf{I t C B N} \mathbf{N a t}_{\mathbf{N a}} \cdot f_{1} \cdot f_{2} \cdot\langle n\rangle\right) \cdot \vec{c} \\
& P(n) \stackrel{2.3}{\approx} f_{2} \cdot\langle(\mathbf{I t}(x, F))(n)\rangle \cdot \vec{c} \\
& \stackrel{(2)}{\approx} \quad\langle F((\mathbf{I t}(x, F))(n))\rangle \cdot \vec{c} \\
& =\left\langle F\left(F^{n}(x)\right)\right\rangle \cdot \vec{c} \\
& =\left\langle F^{n+1}(x)\right\rangle \cdot \vec{c} \\
& =\langle(\mathbf{I t}(x, F))(n+1)\rangle \cdot \vec{c}
\end{aligned}
$$

In the proof, we say that $f_{2} \cdot\left(\mathbf{I t C B} \mathbf{N}_{\mathbf{N a t} \rightarrow B} \cdot f_{1} \cdot f_{2} \cdot\langle n\rangle\right) \cdot \vec{c} \approx f_{2} \cdot\langle(\mathbf{I t}(x, F))(n)\rangle . \vec{c}$. This may may not be immediately obvious, as the sub-term $\mathbf{I t C B} \mathbf{N}_{\mathbf{N a t} \rightarrow B} \cdot f_{1} \cdot f_{2} \cdot\langle n\rangle$ is incomplete. However, it is an immediate consequence of Lemma 2.3. If $M, N$ are terms of arity $k$, and $M . t_{1} \ldots . t_{k}={ }_{p} N . t_{1} \ldots . t_{k}$ for all $\vec{t}$, then $M \approx N$. 